Canad. Math. Bull. Vol. 19 (3), 1976

\title{
A NOTE ON BANACH SPACES CONTAINING $\ell_{1}$
}

\author{
BY \\ ROBERT H. LOHMAN
}

\begin{abstract}
If $Y$ is a subspace of a Banach space $X$, either $Y$ contains an isomorphic copy of $\ell_{1}$ or each weak Cauchy sequence in $X / Y$ has a subsequence that is the image under the quotient mapping of a weak Cauchy sequence in $X$. If $X$ is weakly sequentially complete and $Y$ is reflexive, $X / Y$ is weakly sequentially complete. Related structural results are given.
\end{abstract}

It has recently been shown that if a Banach space $X$ contains a bounded sequence $\left(x_{n}\right)$ having no weak Cauchy subsequence, then $\left(x_{n}\right)$ has a subsequence that is basic and equivalent to the unit vector basis of $\ell_{1}$. A proof of this theorem for real scalars can be found in [5], while a proof for the complex case is given in [2]. The purpose of this note is to show how this result can be applied in the study of the structure of Banach spaces. The letters $X, Y, Z$ will denote Banach spaces over the real or complex scalars. The term "subspace" will mean "closed, infinite-dimensional, linear subspace." Recall that $X$ is weakly sequentially complete if every weak Cauchy sequence in $X$ converges weakly to an element of $X . X$ has the Schur property if weak and norm convergence of sequences coincide in $X$. If $X$ has the Schur property, then a weak Cauchy sequence in $X$ is norm convergent.

If $\left(x_{n}\right)$ is a sequence in $X,\left[x_{n}\right]$ denotes its closed linear span in $X$. For terminology and properties related to Schauder bases, the reader is referred to [6]. If $\left(x_{n}\right)$ and $\left(y_{n}\right)$ are bases for $X$ and $Y$, respectively, they are said to be equivalent provided $\sum_{n=1}^{\infty} a_{n} x_{n}$ converges if and only if $\sum_{n=1}^{\infty} a_{n} y_{n}$ converges. This is equivalent to the existence of an isomorphism $T$ of $X$ onto $Y$ satisfying $T x_{n}=y_{n}$ for all $n$.

THEOREM 1. Let $Y$ be a subspace of $X$ and let $Q: X \rightarrow X / Y$ denote the quotient mapping. Then either $Y$ contains an isomorphic copy of $\ell_{1}$ or each weak Cauchy sequence in $X / Y$ has a subsequence that is the image under $Q$ of a weak Cauchy sequence in $X$.

Proof. Assume that $\left(u_{n}\right)$ is a weak Cauchy sequence in $X / Y$ such that no

Received by the editors September 10, 1975 .

AMS 1970 Subject Classification. 46B15, 46A35. Key Words and Phrases. Banach space. weakly sequentially complete, Schur property. 
subsequence of $\left(u_{n}\right)$ is the image under $Q$ of a weak Cauchy sequence in $X$. Choose a bounded sequence $\left(x_{n}\right)$ in $X$ such that $Q\left(x_{n}\right)=u_{n}$ for all $n$. Then $\left(x_{n}\right)$ has no weak Cauchy subsequence. By passing to a subsequence, we may assume $\left(x_{n}\right)$ is a basic sequence that is equivalent to the unit vector basis of $\ell_{1}$. Let $v_{n}^{\prime}=u_{2 n}-u_{2 n-1}$ for all $n$. Since $v_{n}^{\prime} \rightarrow 0$ weakly, there is an increasing sequence $\left(p_{n}\right)$ and a sequence $v_{n} \in \operatorname{co}\left\{v_{k}^{\prime}: p_{n} \leq k \leq p_{n+1}-1\right\}, n=1,2, \ldots$, such that $\left\|v_{n}\right\| \rightarrow 0$ (cf. [3, p. 422]). Let $\left(w_{n}\right)$ denote the corresponding sequence of convex combinations far out in $\left(x_{2 n}-x_{2 n-1}\right)$. Note that $\left(x_{2 n}-x_{2 n-1}\right)$ is also equivalent to the unit vector basis $\left(e_{n}\right)$ of $\ell_{1}$, say under an isomorphism $T$ carrying $x_{2 n}-x_{2 n-1}$ into $e_{n}$. Then $T$ carries $\left(w_{n}\right)$ into a block basis $\left(z_{n}\right)$ of $\left(e_{n}\right)$, where each $z_{n}$ is a convex combination of $e_{k}$, for $p_{n} \leq k \leq p_{n+1}-1$. Therefore, $\left\|z_{n}\right\|=1$ for all $n$, and it follows by Lemma 1 of [4] that $\left(z_{n}\right)$, and hence $\left(w_{n}\right)$, is equivalent to $\left(e_{n}\right)$. Moreover, $Q\left(w_{n}\right)=v_{n}$ for all $n$. If $\left(w_{n}^{*}\right)$ denotes the sequence of coefficient functionals in $\left[w_{n}\right]^{*}$, we have $M=\sup _{n}\left\|w_{n}^{*}\right\|<\infty$. Since $d\left(w_{n}, Y\right) \rightarrow 0$, there exists an increasing sequence $\left(n_{k}\right)$ of positive integers and a sequence $\left(y_{k}\right) \subset Y$ such that $\left\|w_{n_{k}}-y_{k}\right\|<M^{-1} 2^{-k-1}$ for all $k$. Then $\sum_{k=1}^{\infty}\left\|w_{n_{k}}^{*}\right\|\left\|w_{n_{k}}-y_{k}\right\|<1$ which, by [1], implies that $\left(w_{n_{k}}\right)$ and $\left(y_{k}\right)$ are equivalent basic sequences. Thus $\left(y_{k}\right)$ is equivalent to the unit vector basis of $\ell_{1}$, completing the proof.

COROLlaRY 2. If $X$ is a weakly sequentially complete Banach space and $Y$ is a reflexive subspace of $X$, then $X / Y$ is weakly sequentially complete.

The criterion for a Banach space to contain $\ell_{1}$ can be used to give the following general property of linear operators.

THEOREM 3. Let $T: X \rightarrow Y$ be a continuous linear operator. Then either $T$ maps bounded sequences into sequences having a weak Cauchy subsequence or there exists a subspace $Z$ of $X$ that is isomorphic to $\ell_{1}$ for which $\left.T\right|_{Z}$ is an isomorphism.

Proof. If $\left(x_{n}\right)$ is a bounded sequence in $X$ such that $\left(T x_{n}\right)$ has no weak Cauchy subsequence, there exists a basic subsequence $\left(T x_{n_{k}}\right)$ equivalent to the unit vector basis of $\ell_{1}$. Thus there is a constant $M>0$ such that for all $m$ and scalars $a_{1}, \ldots, a_{m}$, we have

$$
M \sum_{k=1}^{m}\left|a_{k}\right| \leq\left\|\sum_{k=1}^{m} a_{k} T x_{n_{k}}\right\| \leq\|T\| \sup _{n}\left\|x_{n}\right\| \sum_{k=1}^{m}\left|a_{k}\right| .
$$

If $Z=\left[x_{n_{k}}\right]$, it follows that $Z$ is isomorphic to $\ell_{1}$ and that $\left.T\right|_{Z}$ is an isomorphism.

Corollary 4. Let $Y$ be a Banach space with the Schur property and let $T: X \rightarrow Y$ be a continuous linear operator. Then either $T$ is compact or there exists a subspace $Z$ of $X$ that is isomorphic to $\ell_{1}$ for which $\left.T\right|_{Z}$ is an isomorphism. 


\section{REFERENCES}

1. C. Bessaga and A. Pelczynski, On bases and unconditional convergence of series in Banach spaces. Studia Math., 17 (1958), 151-164.

2. L. Dor, On sequences spanning a complex $\ell_{1}$ space, Proc. Amer. Math. Soc., 47 (1975), 515-516.

3. N. Dunford and J. Schwartz, Linear Operators Part I. Interscience: New York, 1958.

4. A. Pelczynski, Projections in certain Banach spaces, Studia Math., 19 (1960), 209-228.

5. H. P. Rosenthal, A characterization of Banach spaces containing $\ell_{1}$, Proc. Nat. Acad. Sci. U.S.A., 71 (1974), 2411-2413.

6. I. Singer, Bases in Banach Spaces I. Springer-Verlag, Berlin, 1970.

\section{DEPARTMENT OF MATHEMATICS}

Kent STATE University

KENT, OH 44242

U.S.A. 